H I G H L I G H T S

GENOME EVOLUTION

\title{
Long way for a shortcut
}

The easiest way to create new genes is to duplicate the ones that are already there, and the quickest way of doing this for many of them is to duplicate the whole genome. This can then be shrunk back down to its previous size while sporting a new collection of genes. This simple theory, which Susumo Ohno proposed more than 30 years ago, has now been validated in yeast by 2 groups that have compared the genome of the most commonly studied member of this taxon, Saccharomyces cerevisiae, with that of its two close relatives.

Finding conclusive evidence of whole-genome duplication (WGD) in S. cerevisiae has been a fruitless task, as most duplicated regions have diverged beyond recognition. What is needed is a yeast species

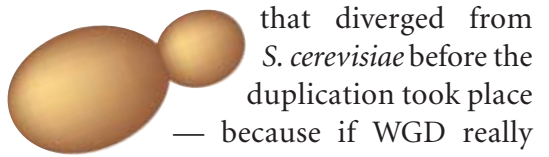
occurred, any one region in this species would have two corresponding regions in S. cerevisiae. As one of almost any two duplicated genes in S. cerevisiae has been lost as the genome returned to its pre-duplication size, regions of homology for each ancestral segment would alternate between the two copies in S.cerevisiae. This pattern is exactly what was seen in the genomes of the yeasts Kluyveromyces waltii and Ashbya gossypii, which were sequenced and analysed by Manolis Kellis and coworkers and by Fred Dietrich and colleagues, respectively.

Kellis et al. identified 253 blocks in $K$. waltii that could each be matched to two regions in the $S$. cerevisiae genome. These blocks - which tiled $85 \%$ of the K. waltii genome and were spread across all

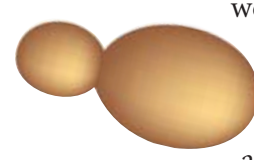

chromosomes - were then used to match up the long soughtafter sister regions in S. cerevisiae that evolution had caused to drift apart. A total of 145 such sister blocks were found, corresponding to 457 gene pairs. So here is the first conclusive evidence that WGD occurred in yeast - but what happened next? Of the 457 gene pairs, $17 \%$ evolved more quickly than their counterparts in K. waltii, and the fact that most cases of accelerated evolution involved only one of the gene duplicates supports the popular idea that duplication leaves one gene copy free to diverge.

The sequencing and genome annotation of A. gossypii by Dietrich et al. led to the same conclusion. Ninety-five percent of all open reading frames in A. gossypii have homologues in S. cerevisiae and most of these are arranged in a conserved linear order. These syntenic regions were used to build a detailed map of ancient S. cerevisiae gene order that also provided definitive proof for the occurrence of WGD in the S. cerevisiae lineage after it split from its common ancestor with A. gossypii.

This work has settled the longstanding controversy over how to interpret the presence of duplicated blocks in the S. cerevisiae genome: are they evidence of WGD or were they caused by local duplications? Although Ohno's idea was an appeal-

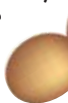

ing one, it didn't necessarily describe the most plausible means of evolutionary innovation - after all, WGD takes the genome for a rather rocky ride. Now that this case has finally been closed, researchers can turn their attention to resolving similar issues in vertebrates, where the debate is still alive.

Tanita Casci

\section{(4) References and links} ORIGINAL RESEARCH PAPERS Kellis, M. et al. Proof and evolutionary analysis of ancient genome duplication in the yeast Saccharomyces cerevisiae. Nature 7 Mar 2004

(doi:10.1038/nature0224)| Dietrich, F. S. etal. The Ashbya gossypii genome as a tool for mapping the ancient Saccharomyces genome. Science $4 \mathrm{Mar}$ 2004 (doi: 10.1126/science.1095781)

\section{IN BRIEF}

\section{EVOLUTION}

Dispersed male networks in western gorillas.

Bradley, B. J. et al. Curr. Biol. 14, 510-513 (2004)

Kin-selection theory, which is used to explain in evolutionary terms why individuals tend to favour relatives, has rarely been seen as relevant to interactions among social groups. Bradley and her colleagues typed groups of western gorillas at ten microsatellite loci. Single males leading social groups were usually related to nearby males. So, kin-selection might explain why interactions between males from different groups are often non-aggressive and why male western gorillas (and humans) tend to remain near to where they were born.

\section{PLANT GENETICS}

The wheat VRN2 gene is a flowering repressor downregulated by vernalisation.

Yan, L. et al. Science 303, 1640-1644 (2004)

Prolonged exposure to low temperatures (vernalization) is required to prompt flowering after winter in wheat varieties that are planted in autumn. VRN2 is the key gene that represses flowering until spring in such varieties. Yan et al. mapped $V R N 2$ in wheat to a region that contained three candidate genes. Confirmation that one of these (ZCCT1) was VRN2 came from wheat and barley strains in which its expression was absent or knocked-down: these strains do not require vernalization for flowering.

\section{DEVELOPMENTAL GENETICS}

\section{Control of fruit patterning by INDEHISCENT.}

Liljegren, S. L. \& Roeder, A. H. et al. Cell 116, 843-853 (2004)

The Arabidopsis thaliana fruit grows after fertilization and encases the growing seeds. The seeds are dispersed when the seedpod opens and a spring-loaded mechanism scatters them. This paper reports the characterization of the INDEHISCENT (IND) gene, which was identified in a genetic screen for mutants that affect seed dispersal and that encode an atypical bHLH protein. IND is required for patterning the three cell types that are needed for fruit opening, which was previously believed to directly require the gene FRUITFULL

\section{TECHNOLOGY}

\section{Controlling transgene expression to study Xenopus} laevis metamorphosis.

Das, B. \& Brown, D. D. Proc. Natl Acad. Sci USA 101, 4839-4842 (2004)

To extend the application of sperm-mediated trangenesis in Xenopus laevis, Das and Brown have used two well-known binaryinducible gene-expression systems (one controlled by doxycyclin and the other by a progesterone analogue). X. laevis that carry inducible lethal transgenes can now be grown to maturity and bred to produce $\mathrm{F}_{1}$ progeny; the transgenes can then be induced when required. 